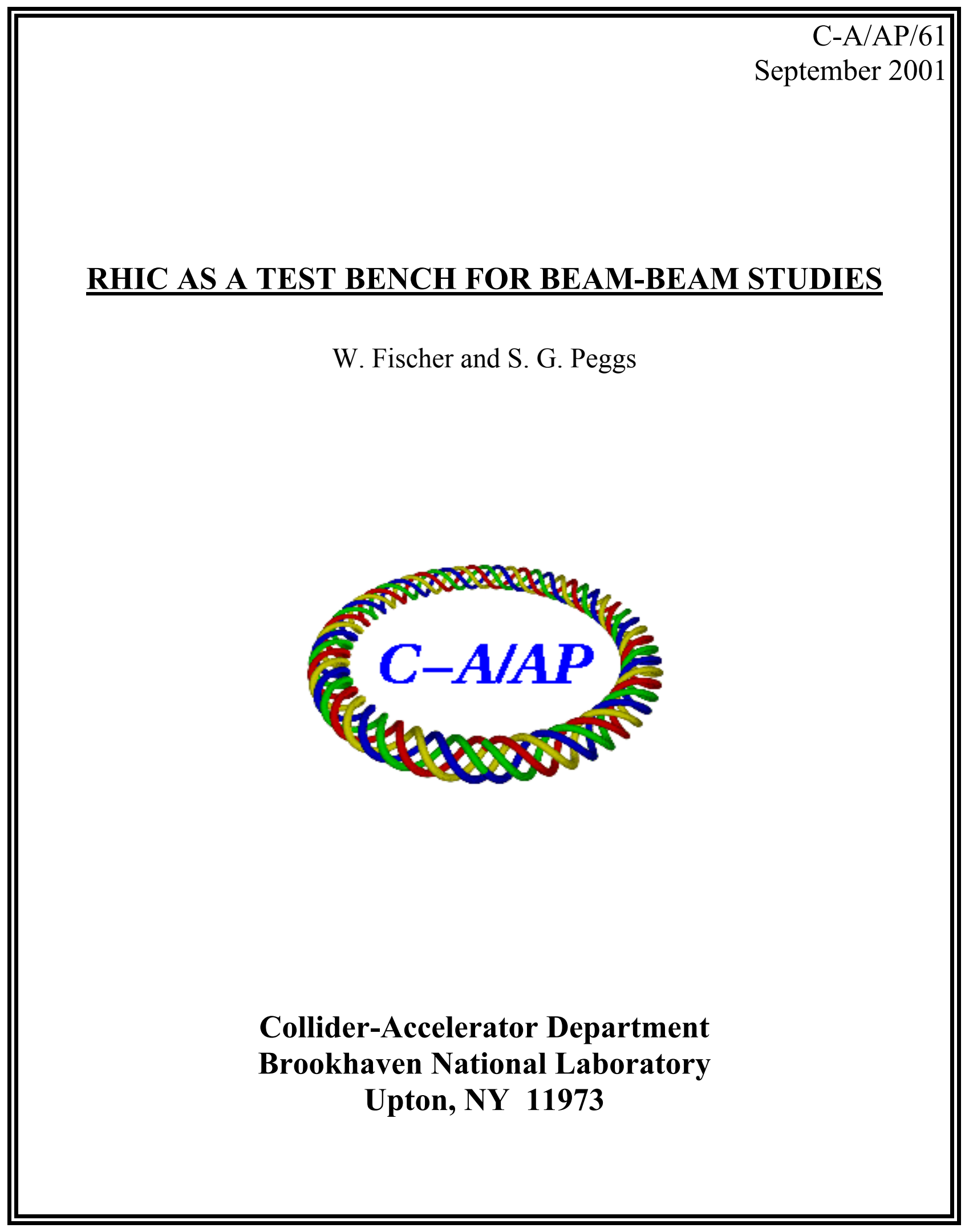




\title{
RHIC AS A TEST BENCH FOR BEAM-BEAM STUDIES *
}

\author{
W. Fischer and S. Peggs, Brookhaven National Laboratory, Upton, NY 11973, USA
}

\section{Abstract}

The Relativistic Heavy Ion Collider (RHIC) is the only existing hadron collider where strong-strong beam-beam effects may occur. It is therefore a good test bench for future hadron colliders for which these effects are relevant. RHIC now approaches its design parameters and its instrumentation is sufficiently developed to allow for beam-beam experiments.

\section{INTRODUCTION}

The Relativistic Heavy Ion Collider (RHIC) went into operation in 2000. The machine has six interaction points (IPs) and supports currently four experiments (see Fig. 1). With gold beams, $10 \%$ of the design luminosity was reached in 2000 [1] and more than $25 \%$ in 2001 . The main machine parameters are summarized in Tab. 1 (a complete list can be found in Ref. [2]).

Beams of equal species collide nominally without a crossing angle. The beams are split horizontally by dipoles (DX) about 10m from the interaction point (see Fig. 2). With 120 or less bunches, symmetrically filled, there are no parasitic beam-beam crossings. Because of this, only a limited number of bunches in both rings are coupled together through beam-beam interactions. With six interaction points, a group of 3 symmetrically distributed bunches in one ring is coupled to a group of of 3 bunches in the

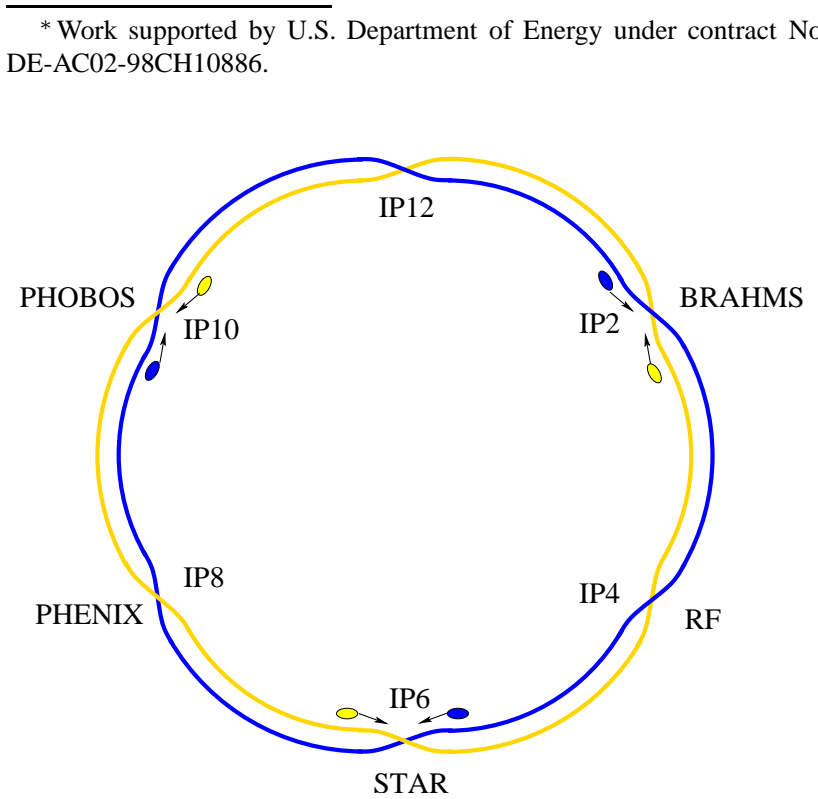

Figure 1: RHIC layout. In stores, only 6 bunches, 3 symmetrically distributed in each ring, are coupled together through the beam-beam interaction.

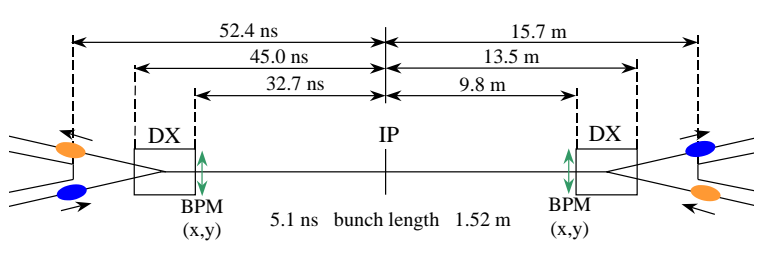

Figure 2: RHIC interaction region layout. The bunches shown correspond to a fill pattern of 120 equally spaced bunches.

other ring, also symmetrically distributed. Every bunch of one group interacts with every bunch of the other group. This can be inferred from Fig. 1. If there is a small difference between the radio frequencies of both rings (see below), all bunches can be coupled together by beam-beam interactions.

When two identical Gaussian beams collide, the horizontal and vertical beam-beam parameters are given by

$$
\xi_{x, y}=\frac{r}{2 \pi \gamma} \frac{N_{b} \beta_{x, y}}{\sigma_{x, y}\left(\sigma_{x}+\sigma_{y}\right)}
$$

where $N_{b}$ is the single bunch population, the classical radius $r$ is $r_{p}=1.5347 \times 10^{-18}$ meters for protons and $r_{A u}=48.992 \times 10^{-18}$ meters for gold. $\beta_{x, y}$ is the beta function, $\sigma_{x, y}$ the transverse rms beam size, and $\gamma$ is the Lorentz factor. Assuming round beams $\left(\beta_{x}=\beta_{y}=\beta_{t}\right)$, $\left(\sigma_{x}=\sigma_{y}=\sigma\right)$ and using as definition for the normalized emittance

$$
\epsilon_{N}=(\beta \gamma) \frac{6 \sigma^{2}}{\beta_{t}}
$$

Eq. (1) can be written as

$$
\xi=\frac{3 N_{b} r}{2 \pi \epsilon_{N}}
$$

Note that the beam-beam parameter is independent of en$\operatorname{ergy}(\gamma)$, and independent of the lattice function $\beta_{t}$. The tune shift of small amplitude particles due to each collision is equal to the parameter $\xi$ no matter what the azimuthal location of the collision, if the beams are round and if they collide head-on. Expected beam-beam parameters are listed in Tab. 1. It may be convenient to parameterize the gold and proton beam-beam parameters as

$$
\xi_{A u}=0.0023 \frac{N_{b}}{10^{9}} \frac{10 \mu \mathrm{m}}{\epsilon_{N}}
$$

and

$$
\xi_{p}=0.0074 \frac{N_{b}}{10^{11}} \frac{10 \mu \mathrm{m}}{\epsilon_{N}} .
$$


Table 1: RHIC principal machine parameters. The column labeled Au2001 shows machine parameters that were achieved with gold beams. The columns labeled A2001+ and p2001+ show machine parameters expected to be approached in the foreseeable future with gold and polarized proton beams respectively.

\begin{tabular}{lccccccc}
\hline \hline parameter & symbol & unit & Au design & Au2001 & Au2001+ & p design & p2001+ \\
\hline collisions per turn & $\ldots$ & $\ldots$ & $2-6$ & $2-6$ & $2-6$ & $2-6$ & $2-6$ \\
kinetic energy range & $E$ & $\mathrm{GeV} / \mathrm{u}$ & $10-100$ & $9-100$ & $9-100$ & $25-250$ & $25-250$ \\
harmonic number & $h$ & $\ldots$ & $7 \times 360$ & 360 & $7 \times 360$ & $7 \times 360$ & $7 \times 360$ \\
gap voltage & $V$ & $\mathrm{MV}$ & 6.0 & 0.3 & 3.0 & 6.0 & 3.0 \\
number of bunches & $N$ & $\ldots$ & 60 & 56 & 110 & 60 & 110 \\
ions per bunch & $N_{b}$ & $\ldots$ & $10^{9}$ & $0.5 \cdot 10^{9}$ & $10^{9}$ & $10^{11}$ & $10^{11}$ \\
normalized emittance $(95 \%)^{\dagger}$ & $\epsilon_{N} x, y$ & $\mu \mathrm{m}$ & 10 & 10 & 10 & 20 & 10 \\
bunch area & $S_{95 \%}$ & $\mathrm{eV} \cdot \mathrm{s} / \mathrm{u}$ & 0.5 & 1.0 & 0.5 & 0.5 & 0.5 \\
average luminosity & $L$ & $\mathrm{~cm}^{-2} \mathrm{~s}^{-1}$ & $2 \cdot 10^{26}$ & $2 \cdot 10^{25}$ & $2 \cdot 10^{26}$ & $10^{31}$ & $2 \cdot 10^{31}$ \\
beam-beam parameter, per IP & $\xi_{x, y}$ & $\ldots$ & 0.0023 & 0.0012 & 0.0023 & 0.0037 & 0.0074 \\
\hline \hline
\end{tabular}

${ }^{\dagger}$ At the beginning of stores.

With $a_{x, y}$ denoting the betatron amplitudes at the IP and defining the variables $\alpha_{x, y}=\left(a_{x, y} / \sigma_{x, y}\right)^{2} / 4$, the weakstrong amplitude dependent tune shift due to head-on collisions can be written as [3]

$$
\begin{aligned}
\Delta Q_{x, y}= & -\xi_{x, y} \int_{0}^{1} d u \exp \left[-\left(\alpha_{x}+\alpha_{y}\right) u\right] \times \\
& \times I_{0}\left(\alpha_{y, x} u\right)\left[I_{0}\left(\alpha_{x, y} u\right)-I_{1}\left(\alpha_{x, y} u\right)\right],
\end{aligned}
$$

where $I_{0}$ and $I_{1}$ denote the modified Bessel functions of zero and first order respectively.

\section{EXPECTED BEAM-BEAM EFFECTS}

\subsection{Weak-Strong Effects}

The beam-beam interaction leads to incoherent and coherent tune shifts [4]. These can can put limitations on the working point. Furthermore, nonlinear resonances may be driven that lead to emittance growth and beam loss. These effects can be further enhanced through nonlinear magnetic field errors and tune modulation. In Fig. 3 a tune footprint is shown for the Au2001+ scenario (see Tab. 1) at the tunes $\left(Q_{x}, Q_{y}\right)=(28.22,29.23)$, which are close to the ones currently used in operation. It is assumed, that beams are colliding in four IPs and are transversely separated in the other two IPs. Sum resonances up to order 9 are added. In Fig. 4 a tune footprint for the p2001+ scenario is shown for collisions at two IPs and transverse separation at the other four IPs. A working point that avoids the resonances is likely to improve the lifetime.

For comparison, significant beam-beam effects are noticed in proton colliders when $\xi=0.004$, with 6 head-on collisions per turn [5]. Increased background rates were observed in the SPS when the tune approached resonances of order 13 and 16 [6].

\subsection{Strong-Strong Effects}

With beams of high and almost equal intensities coherent modes of transverse oscillation ( $\sigma$ and $\pi$ modes) may

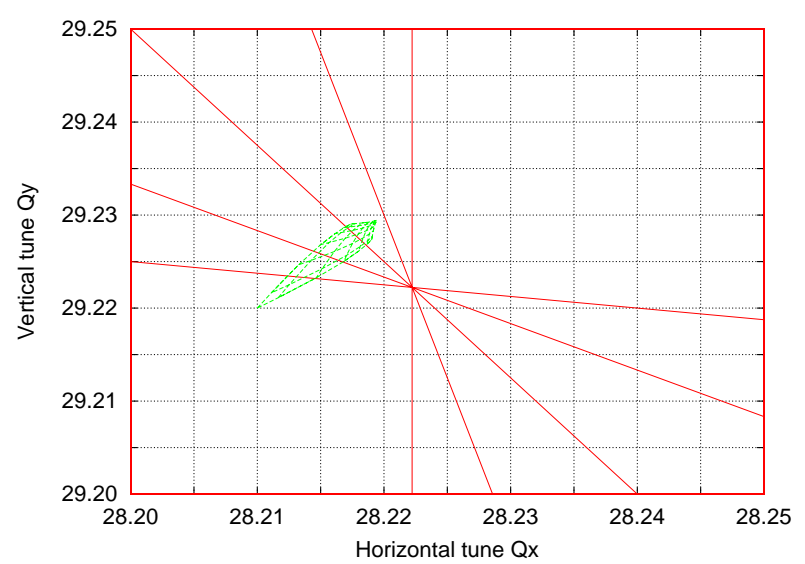

Figure 3: Beam-beam footprint for amplitudes up to $6 \mathrm{rms}$ beam sizes with the Au2001+ parameters and four IPs. The working point is $\left(Q_{x}, Q_{y}\right)=(28.22,29.23)$. Also shown are sum resonances of order 9 .

become visible. The simulation in Ref. [7] clearly shows these modes in the transverse spectra for a single bunch of beam in each ring at the gold design parameters (see Tab. 1). Should the $\pi$ mode be outside the continuum spectrum, it will not be damped.

A simulation in Ref. [8] showed unstable beam centroid oscillations when the beam-beam parameter becomes larger than a critical value, $\xi>\xi_{c}$. The growth rate of the unstable amplitude oscillations is enhanced through nonlinear field errors in the lattice. Furthermore, transverse emittance growth is strongly enhanced under these conditions.

\section{BEAM-BEAM OBSERVATIONS DURING OPERATION}

Up to now colliding beams were only achieved in gold operation. A coherent tune shift of about $10^{-3}$ was measured when beams with $0.3 \times 10^{9}$ ions were brought in and 


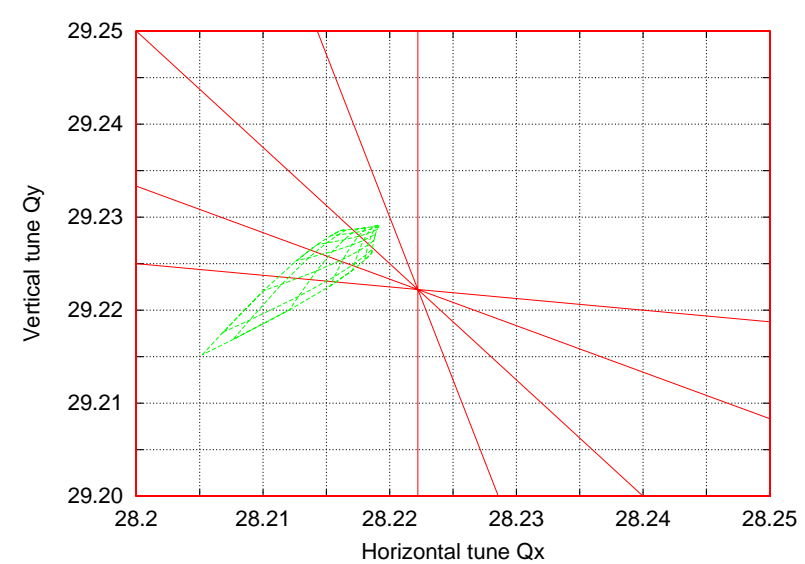

Figure 4: Beam-beam footprint for amplitudes up to $6 \mathrm{rms}$ beam sizes with the p2001+ parameters and two IPs.

out of collision longitudinally. This seems to indicate a relatively minor effect. However, frequently a lifetime deterioration is observed when the beams are brought into collision. Fig. 5 shows such a case at storage energy. Usually the lifetime can be improved by adjusting the general beam conditions such as closed orbit, tune and chromaticity.

In RHIC, the rf systems of both rings are independent since it is planned to accelerate different ion species, which may require different radio frequencies. Thus, the radio frequencies of the Blue and Yellow ring can differ when the phase and radial loops are closed. The small radio frequency difference results in beam-beam collisions with longitudinally moving crossing locations. At injection, this leads reproducibly to lifetime problems in one of the two beams and a scheme was implemented to enforce equal radio frequencies and separate the beams longitudinally [9].

More recently, beam losses along the ramp (when the rf loops are closed and a small difference in the radio frequencies exist) were also attributed to the beam-beam interaction [10], and a transverse separation was implemented in the interaction regions along the ramp to ameliorate the effect [11].

The deterioration of lifetime when both beams have different radio frequencies can be explained by tune modulation that is caused through the longitudinal movement of the interaction point through the interaction region [12]. Typical differences in the radio frequencies lead to tune modulation frequencies of the order of $1 \mathrm{~Hz}$ with modulation depth of up to a few $10^{-3}$.

During stores a transverse emittance growth was observed that is much larger than expectations from intrabeam scattering $[13,14]$, which may be caused by beambeam interactions. Furthermore, there are indications that the transverse emittance growth increases during vernier scans (in which the luminosity is recorded as a function of the transverse beam separation) when the beams collide with a transverse offset $[5,14,15]$.

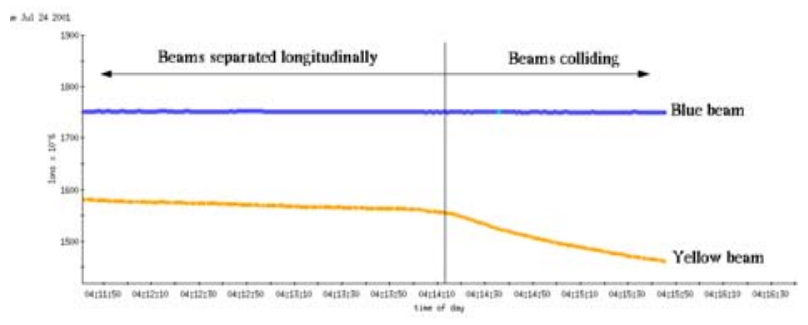

Figure 5: Observed lifetime deterioration due to beambeam effect in gold operation.
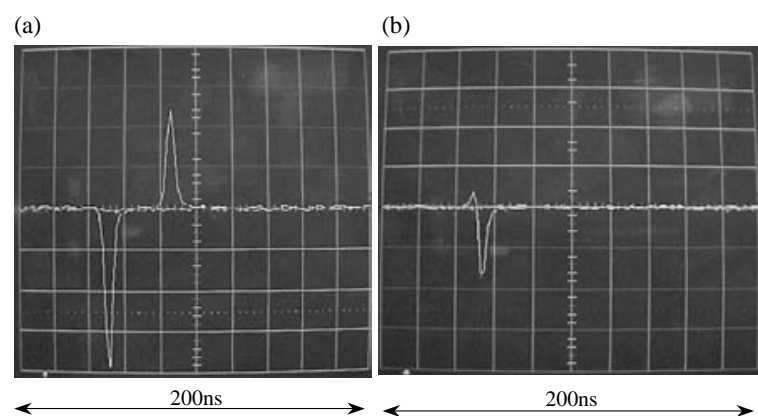

Figure 6: One Blue (upper) and one Yellow (lower) bunch seed with a wall current monitor located at an IP. Due to the different current directions of both beams, the wall current has a positive sign for one and a negative sign for the other beam. In part (a) the beams are not colliding at the IP, in part (b) they do.

\section{POSSIBLE BEAM MANIPULATIONS IN INTERACTION REGIONS}

At storage the beam positions in the interaction regions are manipulated longitudinally and transversely. Longitudinally the beam can be separated or brought into collision (see Fig. 6). The IP can be moved to any location between the crotches (see Fig. 2). By shifting the IP between the DX magnet and the crotch a crossing with up to $90 \mathrm{~mm}$ horizontal separation ( $80 \sigma$ at storage energy for $\epsilon_{N}=10 \mu \mathrm{m}$ and $\beta^{*}=5 \mathrm{~m}$ ) can be achieved. The IP location can be changed in steps of $30 \mathrm{~mm}$. With a small radio frequency difference between the rings the IP can also be shifted continuously. This happens routinely during ramps when both rings run with independent phase and radial loops.

Transversely any of the two beams can be moved laterally in steps of $10 \mu \mathrm{m}$. The crossing angle can be changed in steps of $1 \mu \mathrm{rad}$ (see Fig. 7). The beam movement is observable in the dual plane DX BPMs (see Fig. 2). Transverse separations of more than 100 transverse rms beam sizes and beam-to-beam crossing angles of at least $2 \mathrm{mrad}$ can be implemented [16]. 


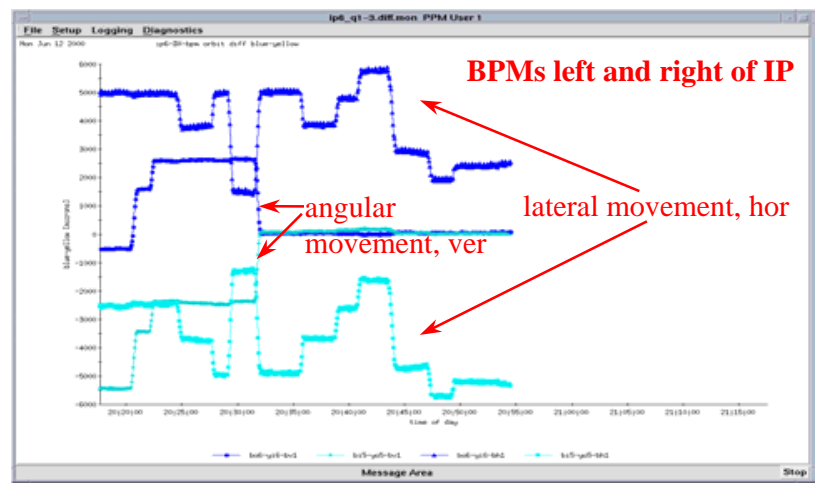

Figure 7: Transverse beam manipulations seen in the DX BPMs. Shown is the angular and lateral movement of the Blue beam at IP6.

\section{INSTRUMENTATION FOR EXPERIMENTS}

The instrumentation for beam observations has been developed and commissioned over the last few years. The current and future capabilities of the main systems are listed below.

Beam Position Monitors In the arcs every quadrupole is equipped with a single plane beam position monitor (BPM). In the interaction regions every quadrupole is equipped with a dual plane beam position monitor. Dual plan BPMs are also located at the inner sides of the beam splitting DX magnets (see Fig. 2) with only a drift space in between. With a trigger signal 128 or 1024 consecutive turns can be read out from every BPM. In the future, two BPMs per plane and ring may deliver $10^{5}$ to $10^{6}$ turns.

Wall Current Monitor Several wall current monitors (WCMs) are available. Typically a full turn can be recorded every $4 \mathrm{~s}$ with a resolution of $0.25 \mathrm{~ns}$ (accelerating buckets are $36 \mathrm{~ns}$, storage buckets $5 \mathrm{~ns}$ long).

Ionization Profile Monitor Horizontal and vertical beam profile monitors are available from ionization profile monitors (IPMs) [22]. Currently profiles are obtained from single bunch stores every $4 \mathrm{~s}$. With more R\&D it may be possible to get profiles from an arbitrary bunch in a regular store (56 or 110 bunches) every 4s. An IPM may also be able to record up to 125000 consecutive turns of a single bunch.

Tune Meters Tunes are available from a system that excites the beam with a small number of small kicks, reads out the beam response in a BPM and computes the Fast Fourier Transform [20]. The resolution of this system is $10^{-4}$. At injection, the tune can also be determined from the spectrum of the injection oscillations. In addition, the tune and tune spread can be measured with a Schottky monitor [21].
Kickers Several kickers are available to excite coherent beam oscillations. The tune kickers [20] can provide a $0.2 \sigma$ horizontal and a $0.1 \sigma$ vertical kick at injection. The injection kicker could provide a vertical kick of more than $5 \sigma$ at injection. At storage energy the kick strength is reduced accordingly.

AC Dipole AC dipoles for both the horizontal and vertical plane will be installed in RHIC. These devices excite coherent dipole oscillation through an AC dipole field running close to the betatron frequencies. With such a resonant drive, in principle, any amplitude can be excited. The AC dipoles can be switched off in about 10 turns to provide free coherent betatron oscillations like through a kicker. The $\mathrm{AC}$ dipoles are located close to an interaction point and shared by both beams.

Pulsed Quadrupole A pulsed quadrupole will be available in the future, also shared by both beams. This quadrupole is intended for transverse echo measurements [24] and would provide a one turn quadrupole kick. The pulsed quadrupole would change the tune by about 0.002 when run continuously at injection.

\section{POSSIBLE BEAM-BEAM EXPERIMENTS}

Beam-beam experiments can be done at injection as well as at storage since the beam-beam parameter $\xi$ is independent of the energy (see Eq. (3)). Experiments at storage energy may be more convenient since orbits are already prepared for collision. However, destructive measurements are best done at injection, since the beam can be restored in a few seconds.

As a basic measurement the coherent tune shift with bunches longitudinally separated or in collision can be determined. Furthermore, the coherent tune shift can be measured as a function of transverse separation, once the bunch intensity is high enough to allow this, given the resolution of the tune measurement. The Schottky system allows to measure the tune and tune spread in the beam. Schottky measurements can also identify beam trapped in resonance islands.

\subsection{Weak-Strong Effects}

To measure weak-strong effects one beam should have a large and one a small intensity. The weaker beam is used for the measurements. In addition to the tune measurements described above, emittance growths can be measured as a function of several parameters such as the tune or the transverse offset. The transverse offset can be made as large as $80 \sigma$ (see above). With IPMs or a scraper, amplitude dependent diffusion may also be determined. 


\subsection{Strong-Strong Effects}

Tune measurements and emittance growth can also be measured with both beams at high intensity. But in addition, coherent modes may be studied. For this only one interaction is desirable with the maximum intensity possible in both beams. A coherent excitation reveals if the $\pi$-mode is damped. In addition, with many-turn BPM observation it can be revealed if the center-of-mass undergoes chaotic motion as predicted in Ref. [8].

\section{SUMMARY}

With RHIC operational and its instrumentation developed, the machine can serve as a test bench for beam-beam effects. Better than any other existing machine, it is suited to investigate strong-strong effects in hadron colliders.

\section{ACKNOWLEDGEMENTS}

The authors wish to thank M. Furman, W. MacKay, W. Herr, S. Tepikian, J. Shi and F. Zimmermann for discussions and help in the preparation of this report.

\section{REFERENCES}

[1] D. Trbojevic, L. Ahrens, M. Blaskiewicz, M. Brennan, M. Bai, P. Cameron, J. Cardona, R. Connolly, J. DeLong, A. Drees, R.P. Fliller, W. Fischer, G. Ganetis, W. Glenn, H. Hahn, T. Hayes, A. Lehrach, W. MacKay, J. Kewisch, S. Peggs, V. Ptitsyn, T. Roser, T. Satogata, T. Shea, K. Smith. S. Tepikain, N. Tsoupas, J. van Zeijts, "Commissioning of the Relativistic Heavy Ion Collider", proceedings of the 2001 Particle Accelerator Conference, Chicago, BNL67969 (2001).

[2] H. Hahn (editor), "RHIC Design Manual", revision of October 2000, http://www.rhichome.bnl.gov/NTshare/rhicdm/00_toc1i.htm.

[3] J.D. Jackson (editor), "Conceptual Design of the Superconducting Super Collider", SSC Central Design Group, SSCSR-2020 (1986).

[4] E. Keil, "Beam-beam Dynamics", CERN Accelerator School, Rhodes, Greece, CERN 95-06 (1995), CERN SL/94-78 (1994).

[5] D. Siergiej,"Beam-Beam Interaction Effects in the Fermilab Collider", PhD thesis, University of New Mexico (1995).

[6] W. Herr, these proceedings.

[7] M.A. Furman, "Beam-Beam Simulations for Separated Beams", proceedings of the US-LHC Collaboration Meeting on Accelerator Physics Experiments for Future Hadron Colliders, Upton, New York, BNL-52601; LBNL-4563, CBP Note 333 (2000).

[8] J. Shi and D. Yao, "Collective Beam-Beam Effects in Hadron Colliders”, Phys. Rev. E, Vol. 62, Number 1, p. 1258 (2000).

[9] J. DeLong, J.M. Brennan, private communication (2001).

[10] A. Drees, D. Trbojevic, "RHIC Logbook" 18 August 2001.

[11] V. Ptitsyn, private communication (2001).
[12] W. Fischer, "Tune Modulation from Beam-Beam Interaction and Unequal Radio Frequencies in RHIC", unpublished note (2001).

[13] W. Fischer, A. Drees, J.M. Brennan, R. Connolly, R. Fliller, S. Tepikian, and J. van Zeijts, "Beam Lifetime and Emittance Growth Measurements of Gold Beams in RHIC at Storage", proceedings of the 2001 Particle Accelerator Conference, Chicago, BNL-67975 (2001).

[14] A. Drees, Z. Xu, "Results from Luminosity Scans during the RHIC 2000 Run", proceedings of the 2001 Particle Accelerator Conference, Chicago (2001).

[15] A. Drees, private communication (2001).

[16] After a problem in one of the IP2 DX magnets was discovered its current was reduced and a beam-to-beam crossing angle of $1.81 \mathrm{mrad}$ implemented temporarily.

[17] S. Peggs, "Parasitic Beam-Beam Collisions and Crossing Angles in RHIC”, BNL RHIC/AP/68 (1995).

[18] S. Peggs, "Beam-beam Collisions and Crossing Angles in RHIC", proceedings of the 1999 LHC Beam-Beam Workshop at CERN, BNL RHIC/AP/169 (1999).

[19] T. Satogata and S. Peggs, "Hadron Beam-Beam Diffusion in 2.5-D", proceedings of the 1999 LHC Beam-Beam Workshop at CERN, BNL RHIC/AP/170 (1999).

[20] P. Cameron, R. Connolly, A. Drees, T. Ryan, H. Schmickler, T. Shea, D. Trbojevic, "ARTUS: A Rhic TUne monitor System", BNL RHIC/AP/156 (1998).

[21] P. Cameron, J. Cupolo, C. Degen, L. Hammons, M. Kesselman, R. Lee, A. Meyer, R. Sikora, "Schottky Measurements During RHIC 2000", proceedings of the 2001 Particle Accelerator Conference, Chicago (2001).

[22] R. Connolly, P. Cameron, R. Michnoff, S. Tepikian, "Performance of the RHIC IPM", proceedings of the 2001 Particle Accelerator Conference, Chicago (2001).

[23] M. Bai, M. Meth, C. Pai, B. Parker, S. Peggs, T. Roser, R. Sanders, D. Trbojevic, A. Zaltsman, "RHIC AC Dipole Design and Construction", proceedings of the 2001 Particle Accelerator Conference, Chicago (2001).

[24] W. Fischer, B. Parker and O. Brüning, "Transverse Echos in RHIC", proceedings of the US-LHC Collaboration Meeting on Accelerator Physics Experiments for Future Hadron Colliders, Upton, New York, BNL-52601 (2000).

[25] W. Fischer and F. Zimmermann, "RHIC Beam Experiments for the LHC Era”, BNL RHIC/AP/179 (1999). 